\section{Ueber den Geruchsinn und die wichtigsten Riechstoffe.}

Von Dr. Ernst Erdmann.

Der Angriff von Herrn Heinrich Walbaum in dieser Zeitschrift 1900 Seite 419 nöthigt mich, ihm auch an dieser Stelle ${ }^{1}$ ) seinen Irrthum nachzuweisen.

Herr Walbaum sagt: „Der Anthranilsäuremethylester wurde von mir im Jahre 1894 als ein Bestandtheil des Neroliöles aufgefunden. Als Beleg wird der Bericht von Schimmel \& Co., Aprilheft 1895 S. 72 angegeben. Ich constatire hiermit, dass sich an der citirten Stelle weder der Name des Herrn Walbaum noch das Wort Anthranilsäuremethylester befindet.

Zur Berichtigung der weiteren Behauptungen von Herrn Walbaum habe ich zu bemerken:

1. Im Jahre 1897 war mir das Vorkommen von Anthranilsäuremethylester im südfranzösischen Orangenblüthenöle aus eigener Untersuchung längst bekannt. Für die gegentheilige unbegründete Annahme Walbaum's ist der Wunsch Vater des Gedankens gewesen ${ }^{2}$ ).

2. Die erste zur Veröffentlichung bestimmte Mittheilung der Entdeckung findet sich nicht im Berichte von $\mathrm{Schimmel} \& \mathrm{Co}$. vom 1. April 1899, sondern in der vom deutschen Patentamte ansgelegten Anmeldung E 5958 vom 28. Mai $\left.1898^{3}\right)$.
Es heisst dort wörtlich:

„Bei der Untersuchung des südfranzösischen Orangenblüthenöles (Néroli Pétales, Nóroli Bigarade) haben wir einen noch nicht bekannten, aber für den Geruch und die Eigenschaften dieses ätherischen Öles charakteristischen stickstoffhaltigen Bestandtheil aufgefunden: es ist dies der Anthranilsäuremethylester. Derselbe ist aus dem Neroliöl so zu isoliren, dass die bei $10 \mathrm{~mm}$ Druck um $125^{\circ}$ siedende Fraction mit wasserfreiem Äther verdünnt und trockenes Salzsäuregas eingeleitet wird; es fällt dann in Krystallnadeln das salzsaure $\mathrm{Salz}$ einer primären Base aus, welche mit dem aus Anthranilsäure synthetisch hergestellten Anthranilsäuremethylester identisch ist. Selbst in dem nicht fractionirten Öl lässt sich die Anwesenheit des Amidoesters dadurch nachweisen, dass man dasselbe mit Salzsänre schüttelt, die saure Lösung diazotirt und mit einem Phenol, z. B. Naphtoldisulfosäure R, zu einem in orangegelben Flocken ausfallendem Farbstoffe combinirt.

Die starke, bisher nicht zu erklärende blaue Fluorescenz des Bigarade-Öles hat in diesem Gehalt an Anthranilsäuremethylester ihreUrsache." . .

3. Die von Schimmel \& Co. in ihrem Aprilberichte über Orangenblüthenöl gemachte Mittheilung ${ }^{4}$ ) ebenso wie die Publication Walbaums $\mathrm{s}^{5}$ ) war eine directe Folge der von meinem Bruder und mir begonnenen Veröffentlichungen über diesen Gegenstand, speciell eine Folge der Auslegung unserer Patentanmeldung auf Darstellung von Anthranilsäuremethylester ${ }^{6}$ ).

\title{
Referate.
}

\section{Organische Chemie.}

\section{F. Cross, E. J. Bevan und Th. Heiberg. Ein- wirkung ron Wasserstoffisuperoxyd anf un- gesättigte Kohlenwasserstoffe. (Berichte 33, 2015.)}

Lässt man Wasserstoffsuperoxyd bei Gegenwart von Eisensulfat auf Acetylen einwirken, so bildet sich Alkohol, Aldehyd und Essigsäure.

Benzol reagirt mit Wasserstoffsuperoxyd und Eisensulfat unter Bildung von Phenol, Brenzcatechin, Hydrochinon and eines sauerstoffhaltigen Productes mit $12 \mathrm{C}$-Atomen. Aus $10 \mathrm{~g}$ Benzol wurden $1,5 \mathrm{~g}$ Phenol und $3,5 \mathrm{~g}$ Brenzcatechin erhalten, Hydrochinon war nur in geringen Mengen entstanden.

1) Wie in den Berichten der deutschen chemischen Gesellschaft 1900 Heft 13.

2) Vgl. Handelsbericht von Sehimmel \& Co., Octoberheft 1897, woselbst es mit Bezug auf meinen Bruder heisst: Wir können nur wünschen, dass die Herrn Erdmann verdriessenden "Zusätze" (zu Neroli, Schimmel \& Co.) ihm noch recht lange "geheimnissvoll" bleiben möchten". - Dieser Wunsch kam zu spüt.

3) Ernst Erdmann und Hugo Erdmann. Darstellung synthetischer Blumengerüche unter Verwendung von Anthranilsäuremethylester.
W. Dickmann. Ueber das Verhalten von Phenyli-cyanat gegen Acetessigester. (Berichte B3, 2002.)

Man hat bisher angenommen, dass Phenylcyanat ein zur Diagnose tautomerer Verbindungen geeignetes Reagens sei, insofern als es nur mit den Enolformen, nicht aber mit den Ketoverbindungen Derivate lieferte, welche die Gruppe: $\mathrm{CH}$. O . CO . $\mathrm{NH} \mathrm{C}_{6} \mathrm{H}_{5}$ enthalten. Verf. hat versucht, in gleicher Weise die Constitution des Acetessigesters festznstellen, hat aber das überraschende Resultat gefunden, dass das Cyanat nicht mit der Hydroxylgruppe, sondern mit dem benachbarten Kohlenwasserstoffrest in Reaction tritt. Das Product ist Acetylmalonanilidsäureester

bez.

$$
\mathrm{CH}_{3} \cdot \mathrm{CO} . \mathrm{CH}<\underset{\mathrm{COO} \mathrm{OC}_{2} \mathrm{H}_{5}}{\mathrm{NO} \mathrm{H}_{5}}
$$

$$
\mathrm{CH}_{3} \cdot \mathrm{C}(\mathrm{OH}): \mathrm{C}<\underset{\mathrm{CO} \mathrm{OO} \mathrm{O}_{2} \mathrm{H}_{5}}{\mathrm{CO}}
$$

dessen Identität durch Überführung in Malonanilidsüure zweifellos festgestellt werden konnte. Demnach erscheint es nicht ausgeschlossen, dass auch

4) "Infolge einer von anderer Seite eingereichten Patentanmeldung sehen wir uns veranlusst, folgendes hier zu veröffentlichen". (Schimmel \& Co. Aprilheft 1899 S. 34.)

$\left.{ }^{5}\right)$ Journal für praktische Chemie (2) $\mathbf{6 9}, 350$.

6) Deutsches Reichspatent 110386 , ausgelegt am 20. Februar 1899. 